\title{
VALORACIÓN NUTRICIONAL Y EMISIÓN DE GASES DE ALGUNOS RECURSOS FORRAJEROS DEL TRÓPICO DE ALTURA
}

\section{NUTRITIONAL EVALUATION AND GAS EMISSIONS OF FORAGE RESOURCES OF THE HIGH TROPICS}

\author{
Edmundo Apráez G. ${ }^{1}$; Arturo Gálvez C. ${ }^{1}$; Carlos Jojoa R. ${ }^{2}$
}

Fecha de recepción: Octubre 03 de $2014 \quad$ Fecha de aceptación: Diciembre 04 de 2014

\section{RESUMEN}

Se determinó la producción de gases (metano, AGVs) en algunos forrajes herbáceos, arbóreos y arbustivos de la región lechera del municipio de Pasto, en altitudes comprendidas entre 2600 a 3200 m.s.n.m. Para ello, se tomaron veinte muestras de praderas, entre monocultivo y mezcla, y de otras plantas que utilizan como alimento del ganado, entre los que se destacan: Holcus lanatus L., Dactylis glomerata L., Trifolium pratense L., Trifolium repens L., Pennisetum clandestinum, Lolium sp, Taraxacum officinale, Rumex crispus L., Phalaris sp, Plantago major L., Avena sativa L. y Smallanthus pyramidalis. Estas plantas fueron incubadas con heces bovinas frescas, los gases generados por esta fermentación se cuantificó utilizando un transductor de presión conectado a un lector digital. Las mediciones se realizaron a las 3, 6, 12, 24, 48 y 72 horas del proceso. La validación estadística se realizó mediante un modelo de medidas repetidas las medias fueron ajustadas y comparadas según la prueba de Tukey - Kramer. La pradera en mezcla de H. lanatus, Lolium sp., P. clandestinum, y T. repens presentó la mayor producción de gas metano con $560 \mathrm{ml} /$ $\mathrm{Kg}$ MS, y el ensilaje de A. sativa, la menor con $30 \mathrm{ml} / \mathrm{Kg}$ MS. La mayor degradabilidad de materia seca (DMO) se observó en A. sativa con 62,84\%, y la menor en la mezcla de Lolium sp., T. repens con $29,79 \%$. La mayor DMO se dio en la mezcla de P. clandestinum, H. lanatus y T. repens, con $91,34 \%$, y en $P$. major con $82,50 \%$. Los valores más altos de ácido propiónico se observaron en $P$.

1 Profesores tiempo completo,Ph. D. Universidad de Nariño. Pasto. Colombia. eapraez@gmail.com.

2 Profesor hora cátedra, Zootecnista. Universidad de Nariño. Pasto. Colombia. carlos.joja@gmail.com 
major, en Lolium sp., y en la mezcla de H. lanatus, Lolium sp., P. clandestinum, y T. repens con 920, $860,860 \mathrm{ml} / \mathrm{L}$ respectivamente.

Palabras clave: Fermentación, producción de gas, metano, rumiantes, técnica in vitro.

\begin{abstract}
Gas production (methane, AGVs) in herbaceous plants, shrubby tree fodder, and trees of the milkproducing region in the municipality of Pasto, at altitudes between 2600-3200 meters above sea level, was determined. Twenty samples of grassland were collected, between monoculture and grass mixture and other plants that are often used as livestock feed, among: Holcus lanatus L., Dactylis glomerata L., Trifolium pratense L., Trifolium repens L., Pennisetum clandestinum, Lolium sp, Taraxacum officinale, Rumex crispus L., Phalaris sp, Plantago major L., Avena sativa L. y Smallanthus pyramidalis. These plants were incubated with fresh bovine feces, and the amount of gases produced by fermentation was quantified using a pressure transducer connected to a digital reader. Measurements were made at 3, 6, 12, 24, 48, and 72 hours of the process. Statistical validation was performed using a model of repeated measures; means were adjusted and compared according to the Tukey-Kramer test. The grassland mixture of H. lanatus, Lolium sp., Kikuyu, and T. repens showed the highest production of methane gas with $560 \mathrm{ml} / \mathrm{kg} \mathrm{DM}$, while A. sativa silage showed the lowest with $30 \mathrm{ml} / \mathrm{kg}$ DM. The highest dry matterdegradability (DMD) was observed in A. sativa with $62.84 \%$, and the lowest in the mixture of Lolium sp. and T. repens with $29.79 \%$. The highest degradability of organic matter (DOM) occurred in the mix of P. clandestinum, H. lanatus, and T. repens, with $91.34 \%$, and in P. major with $82.50 \%$. The highest values of propionic acid were observed in P. major, Lolium sp., and in the mixture of H. lanatus, Lolium sp., P, clandestinum, and T. repens, with 920, 860, and $860 \mathrm{ml} / \mathrm{L}$, respectively.
\end{abstract}

Keywords: Fermentation, gas production, methane, ruminants, in vitro technique.

\section{INTRODUCCIÓN}

La ganadería en Colombia, es un renglón cultural y socioeconómico relevante para el desarrollo del agro, especialmente para el Departamento de Nariño, el cual se ha caracterizado por su vocación agrícola. Incrementar la producción de alimentos para una población que se ha duplicado en los últimos 50 años, es un reto ante la panorámica existente. La actividad agrícola se lleva a cabo en gran parte del territorio, extendiéndose notablemente en los últimos años; sin embargo, es fuertemente cuestionada debido al uso irracional del recurso edáfico, hídri- co y ambiental de la región, especialmente en suelos frágiles de ladera en trópico alto, donde es más notable el impacto ambiental causado. La problemática enunciada podría considerar a futuro la creación de políticas que conduzcan a regular el número de bovinos manejados por cada región o buscar la manera de hacerlos más eficientes con base enlos recursos existentes.

Los sistemas de producción de rumiantes implementados en diferentes países del Trópico americano se han caracterizado por la utilización de grandes áreas con potencial para la producción de forraje, empleo de dosis crecientes 
de agroquímicos, así como por el uso elevado de insumos (Álvarez, 2000).La situación anterior ha traído como consecuencia la degradación de praderas y contaminación de las cuencas de agua, aceleración de la tasa de deforestación e incrementos en la emisión de los gases asociados al calentamiento global (Berra et al., 2006).

Los cambios en el clima del planeta a través del tiempo fueron y son producidos por diversos procesos naturales que afectan al sistema climático o alguno de sus componentes. Desde que en 2006 se publicara el informe de la FAO "Livestock Long Shadow" que afirmaba que el $18 \%$ de los gases de efecto invernadero (GEI) a nivel mundial son consecuencia de la ganadería, se ha producido un bombardeo mediático que ha repercutido en el sector. La ganadería en general y el vacuno en particular, se consideran como la principal especie productora, vía fermentación entérica, de metano (gas con efecto invernadero) y el tratamiento anaeróbico de las excretas ganaderas. Estas últimas son también fuente de óxido nitroso, con efecto invernadero 300 veces superior al $\mathrm{CO}_{2}(\mathrm{FAO}, 2006)$.

La fermentación ruminal de los sustratos nutricios puede ser estudiada por métodos in vivo, in situ e in vitro. En los últimos años, varias técnicas in situ e in vitro han sido desarrolladas, debido a que en los estudios in vivo los alimentos sólo pueden ser evaluados en raciones totales, son difíciles de estandarizar y requieren considerables recursos económicos, técnicos, animales y humanos. La técnica in vitro de uso más frecuente es la descrita por Tilley y Terry (1963) modificada por Goering y Van Soest (1970), para estimar la digestibilidad verdadera de la materia seca (MS). La técnica de producción de gases es otro método in vitro para estimar la extensión y la cinética de degradación de un alimento a través del volumen de gas producido durante el proceso fermentativo (Theodoridou et al., 2012). Una de las ventajas de este procedimiento es que el curso de la fermentación y el papel de los componentes solubles del sustrato pueden ser cuantificados (Pell et al., 1997).

La presente investigación permitió identificar qué tipo y cantidad de gases efecto invernadero emiten las praderas más comúnmente usadas en los sistemas de producción lechera del municipio de Pasto y cuales especies forrajeras permiten reducir las emisiones.

\section{MATERIALES Y MÉTODOS}

La investigación se realizó en la cuenca lechera del municipio de Pasto, departamento de Nariño, en la montaña altoandina colombiana, con alturas entre 2600 a 3200 m.s.n.m, temperatura media de $12^{\circ} \mathrm{C}, 75-80 \%$ de humedad relativa y precipitación pluvial de $600-1100 \mathrm{~mm}$ por año. De acuerdo con Holdridge, corresponde a la zona de vida Bosque Seco Montano Bajo (bsMB) (Holdridge, 1978). Se seleccionaron6 fincas de la cuenca lechera del municipio de Pasto, corregimientos de Catambuco y Gualmatán, donde se muestrearon 20 dietas básicas, entre monocultivos de gramíneas y mezclas herbáceas, arbustos y árboles forrajeros de uso común en la zona de estudio.

Las muestras de heces vía rectal, se tomaron en el Centro de Investigación CORPOICA, ubicado en el kilómetro 5vía al corregimiento de Obonuco. Las muestras fueron procesadas en los Laboratorios Especializadosde la Universidad de Nariño. Se identificó la época óptima de corte o pastoreo, principalmente en la época de prefloración. Las 20 dietas se detallan en la Tabla 1. 
Tabla 1. Recursos forrajeros del trópico de altura evaluados en el estudio

\begin{tabular}{l|c}
\hline \multicolumn{1}{c|}{ Recurso forrajero } & Dieta \\
\hline Saboya Holcus lanatus L., azul orchoro Dactylis glomerata L. trébol rojo Trifolium pratense L. & 1 \\
\hline Kikuyo Pennisetum clandestinum Hochst. ex Chiov sin fertilizar & 2 \\
\hline Aubade Lolium sp., Samson Lolium sp., trébol blanco Trifolium repens L. & 3 \\
\hline Kikuyo fertilizado & 4 \\
\hline Saboya, Kikuyo, diente de león Taraxacum officinale F.H. Wigg, lengua de vaca Rumex crispus L. & 5 \\
\hline Saboya, kikuyo, trebo blanco & 6 \\
\hline Saboya, aubade, kikuyo, trébol rojo & 7 \\
\hline Aubade, Samson, sterling Lolium sp., trébo rojo & 8 \\
\hline Saboya, Aubade, Samson, trébol rojo, diente de león & 9 \\
\hline Samson, sterling & 10 \\
\hline Nui Lolium sp. & 11 \\
\hline Ohau Lolium sp. & 12 \\
\hline Llantén Plantago major L. & 13 \\
\hline Avena cayuse Avena sativa L. & 14 \\
\hline Samson & 15 \\
\hline Saboya, kikuyo, brasilero Phalaris sp., lengua de vaca & 16 \\
\hline Saboya & 17 \\
\hline Kikuyo, Saboya, Trébol blanco (ensilaje) & 18 \\
\hline Avena ensilaje & 19 \\
\hline Colla negra Smallanthus pyramidalis & 20 \\
\hline
\end{tabular}

Para el muestreo de las praderas con especies herbáceas, se siguió la metodología descrita por Sierra (2006) para aforar potreros, mediante el trazo de transectos imaginarios en zigzag, en cuyas direcciones se lanza un marco de $25 \times 25$ $\mathrm{cm}$ hasta recolectar una muestra de 500 gramos y posterior corte del pasto.

Las arbóreas y arbustivas se muestrearon según el método del árbol próximo, utilizado por Smith (1993), que consiste en iniciar las mediciones con un árbol o arbusto cualquiera que esté en el interior de la mancha de árboles y a partir del cual se muestrearon todos aquellos que secuencialmente estuvieron más cerca, con la restricción de no volver a medir aquel que ya se midió. Se muestrearon hojas y tallos tiernos, simulando el ramoneo de los bovinos. De las submuestras se conformó una muestra de 500 gramos para envío al laboratorio.

Composición botánica. Se tomó una muestra de cada una de las 20 praderas con las mezclas descritas, utilizando la metodología de aforos descrita por Sierra (2006). Se obtuvieron 20 muestras de 500 gramos cada una, de donde se determinó la composición botánica por peso de la biomasa.

Análisis bromatológico. Se tomó muestras de los recursos evaluados, que fueron posteriormente secados y molidos para el análisis químico proximal, de acuerdo con los métodos establecidos por la A.O.A.C. (1990), que incluyen 
el contenido de humedad (método 930,04), proteína cruda por el método de Kjeldahl (Nx6.25) (método 955,04), cenizas (por calcinación a $550^{\circ} \mathrm{C}$ ) (método 930,05), extracto etéreo (método 962,09) y fibra bruta (método 920,39), fibra detergente neutra (FDN), fibra detergente ácida (FDA), y espectrofotometría para minerales. La energía bruta (EB) se determinó por medio de bomba calorimétrica, las fracciones de fibra de acuerdo con el método de Goering y Van Soest (1970).

\section{TÉCNICA in vitro DE PRODUCCIÓN DE GASES}

Se trabajó con un total de 67 botellas de incubación, acondicionadas en un acuario con una gradilla en su interior para su estabilidad, 20 dietas con tres réplicas cada uno, 5 blancos que contenían medio de digestión e inóculo fecal, pero sin sustrato, para ajustar la producción de gas debida a los sustratos, y 2 estándares: Desmodium heterocarpon y Brachiaria humidicola donados por el Centro Internacional de Agricultura Tropical (CIAT).

El estudio contempló la colecta de heces mediante la técnica per rectum, a 3 vacas donantes de la raza Holstein mestizas adultas, cuya alimentación base estuvo compuesta por Pennisetum clandestinum y Holcus lanatus en mayor porcentaje.

A través de un transductor de presiones marca Autonics, con lector digital, conectado a una fuente energética constante de 15 voltios (para evitar un cese inesperado de energía eléctrica), se registró la presión generada por la acumulación de gases en el interior de las botellas, efecto de la fermentación. La presión formada al interior de los frascos, fue medida en libras por pulgada cuadrada (PSI). La estimación del volumen de gas a partir de valores de presión, se realizó teniendo en cuenta la ley de los gases de
Boyle y Gay-Lussac, donde: $\mathrm{P}_{\mathrm{G}}=\left(\mathrm{V}_{\mathrm{s}} / \mathrm{P}_{\mathrm{a}}\right) * \mathrm{P}_{\mathrm{t}^{\prime}}$ siendo $\mathrm{P}_{\mathrm{G}}=$ Producción de gases $(\mathrm{ml}) ; \mathrm{V}_{\mathrm{s}}=$ Volumen de gases en la parte superior del frasco $(\mathrm{ml}) ; \mathrm{P}_{\mathrm{a}}=$ Presión atmosférica (psi) y $\mathrm{P}_{\mathrm{t}}=$ Presión obtenida en el transductor (psi). Los horarios de lectura planteados fueron: horas 0,3, 6,12, 24,48 y 72 de incubación, datos que fueron registrados digitalmente para su posterior análisis.

Para la determinación de AGV's, se siguió la metodología descrita por Narváez y Delgado (2011).

El material degradado, se calculó por diferencia de pesos entre los crisoles vacíos, el peso inicial de la muestra incubada y los crisoles con residuo tras el secado en estufa y en mufla respectivamente. La materia seca (MS) degradada se calculó como la diferencia entre el peso de la muestra al inicio de la incubación y el peso del residuo en crisol al final de la incubación.

Los volúmenes de gas obtenidos para cada forraje después del periodo de fermentación, fueron procesados mediante análisis de medidas repetidas en el tiempo (PROC MIXED SAS 9.2) a fin de determinar las diferencias en la producción de gas. Las medias se ajustaron y compararon según la prueba de Tukey - Kramer $(p<0,05$.$) . El$ mismo análisis fue empleado para la evaluación de la degradabilidad de MS y MO.

Los resultados de presión y volumen obtenidos durante el proceso fermentativo, fueron utilizados para establecer una ecuación de regresión utilizando PROC REG también del programa estadístico SAS.

\section{RESULTADOS Y DISCUSIÓN}

La composición botánica de cada una de las dietas de cada finca aparece en la Tabla 2. 
Tabla 2.Composición botánica de las fincas evaluadas

\begin{tabular}{|c|c|}
\hline FINCA & COMPOSICIÓN BOTÁNICA \\
\hline San José (B) & Saboya $60 \%$, azul orchoro $35 \%$, trébol rojo $5 \%$ \\
\hline Los Molinos & Kikuyo sin fertilizar $100 \%$ \\
\hline San José (B) & Aubade $48 \%$, Samson $45 \%$, trébol blanco $7 \%$ \\
\hline San José (B) & Kikuyo fertilizado $100 \%$ \\
\hline Los Molinos & Saboya $60 \%$, Kikuyo $30 \%$, diente de león $5 \%$, lengua de vaca $5 \%$ \\
\hline San José (R) & Saboya $65 \%$, kikuyo $30 \%$, trébol blanco $5 \%$ \\
\hline San José (R) & Saboya $60 \%$, Aubade $25 \%$, kikuyo $10 \%$, trébol rojo $5 \%$ \\
\hline San José (R) & Aubade $30 \%$, Samson 30\%, Sterling 30\%, trébolrojo $10 \%$ \\
\hline San José (R) & Saboya $30 \%$, Aubade $30 \%$, Samson $30 \%$, trébol rojo $5 \%$, diente de león $5 \%$ \\
\hline La Frisona & Samson $50 \%$, Sterling $50 \%$ \\
\hline La Frisona & Nui $100 \%$ \\
\hline La Frisona & Ohau $100 \%$ \\
\hline El Rincón & Llantén $100 \%$ \\
\hline Los Molinos & Avena cayuse $100 \%$ \\
\hline El Rincón & Samson $100 \%$ \\
\hline El Encanto & Saboya $40 \%$, kikuyo $30 \%$, brasilero $20 \%$, lengua de vaca $10 \%$ \\
\hline El Encanto & Saboya $100 \%$ \\
\hline El Encanto & Kikuyo $85 \%$, Saboya $12 \%$, Trébol blanco 3\% (ensilaje) \\
\hline Obonuco & Avena ensilaje $100 \%$ \\
\hline El Rincón & Colla negra $100 \%$ \\
\hline
\end{tabular}

En las praderas evaluadas, se encontró una mayor proporción de Holcu lanatus con un $43,85 \%$, seguido de especies introducidas del genero Lolium sp., como Sterlingy Samson con $40 \%$ y $38,75 \%$ respectivamente. Narváez y Delgado (2011) reportan praderas con el 53,26\% de estas dos especies en un estudio realizado en la finca de Chimangual, de la Universidad de Nariño, ubicada en el distrito lechero de Guachucal. Los resultados encontrados corroboran la relevancia que ocupan las especies foráneas del género Lolium sp. para los sistemas lecheros especializados de trópico alto. Pese al gran porcentaje de especies mejoradas en las praderas, la composición botánica estuvo conformada también por especies naturalizadas como Azul orchoro (Dactyli sglomerata L.), Trébol rojo (Trifolium pratense L.), Trébol blanco (Trifolium repens L.), seguido por Holcus lanatus L., Phalaris sp., Trifolium pratense L. y finalmente algunas arvenses como lengua de vaca Rumex crispus L. y diente de león Traxacum officinale 
F.H. Wigg, especies pertenecientes a la familia Polygonaceae, caracterizadas por un rápido crecimiento, producción constante de biomasa y propagación esporádica, las cuales han sido parte natural de la cobertura del suelo, aportando a la producción de biomasa y la calidad nutritiva de la dieta.

\section{Contenido nutricional}

En la tabla 3, se muestran los resultados bromatológicos de las especies evaluadas

Materia seca (MS). El porcentaje de materia seca, estadísticamente, no registró diferencias $(\mathrm{p}>0,05)$ (Tab. 3), presentándose los valores más altos en colla negra, avena ensilaje, y la pradera en mezcla de Aubade, Samson, Sterling y trébol rojo. Belalcázar y Narváez (2008), reportan valores de $26 \%$ de MS, para la colla, en estudios realizados en las instalaciones de la Universidad de Nariño. Por otro lado es bien sabido que, con el ensilaje de cereales se busca aprovechar el contenido elevado de carbohidratos para ser transformados en ácido láctico, bajo poder tampón y elevado contenido en materia seca (Calsamiglia et al., 2005).
La materia seca, en general, presentó una correlación negativa muy baja (-0.34067) con la producción total de gas metano $\left(\mathrm{CH}_{4}\right)$, Figura 1, aspecto que permite afirmar que no hubo relacióndirecta entre los contenidos de MS en los recursos evaluados y la producción y emisión de $\mathrm{CH}_{4}$ por parte de los animales.

\section{Proteína}

El contenido proteico no presentó diferencias estadísticas $(\mathrm{p}>0,05)$ entre tratamientos; sin embargo, la dieta de pasto Nui Lolium sp. presentó un importante valor numérico, seguido por el Llantén, Samson Lolium sp. y colla negra. Los valores obtenidos en los pastos Nui y Samson pueden estar relacionados con óptimos estados vegetativos de las pasturas al momento del corte, por ser especies introducidas y mejoradas, y una mejor disponibilidad de nutrientes en el suelo, fruto de la aplicación de riego y fertilizantes por parte del ganadero. Adicionalmente, las praderas en mezcla de gramíneas con leguminosas y arvenses reportan valores importantes como en el kikuyo, saboya, brasilero, lengua de vaca, saboya, Samson, Aubade, trébol rojo y diente de león (Tab. 3).

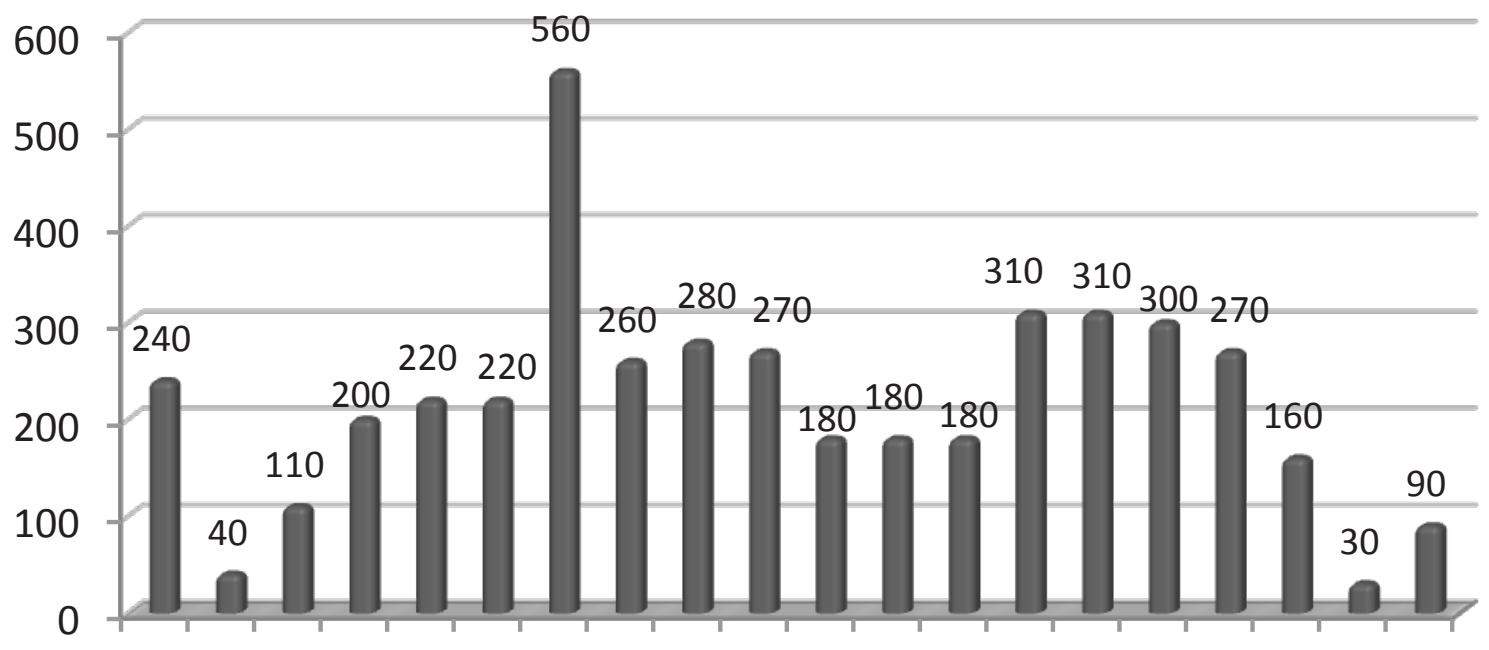

Figura 1. Producción de $\mathrm{CH}_{4}$ por $\mathrm{Kg}$ de MS, los números en el eje $\mathrm{X}$ representan los recursos forrajeros mencionados en la Tabla 3 


\begin{tabular}{|c|c|c|c|c|c|c|c|c|c|c|c|c|c|c|c|c|c|c|c|c|}
\hline$\sum^{\infty} a^{\circ}$ & ڤ̂̀ & ?. & 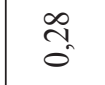 & on & त̂ & \begin{tabular}{l}
\multirow{J}{*}{} \\
o
\end{tabular} & $\begin{array}{l}\hat{n} \\
\tilde{0}\end{array}$ & $\begin{array}{l}\stackrel{\sim}{*} \\
\tilde{\sigma} \\
0\end{array}$ & 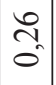 & స్ & $\tilde{c}$ & $\tilde{\tilde{o}}$ & $\begin{array}{c}2 \\
\tilde{0} \\
0\end{array}$ & $\left|\begin{array}{l}\infty \\
0 \\
0\end{array}\right|$ & $\begin{array}{l}\partial \\
\dot{0} \\
0\end{array}$ & $\overrightarrow{0}$ & $\overrightarrow{0}$ & त̂. & 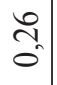 & fo \\
\hline $20^{\circ}$ & $\underset{0}{\mathbb{J}}$ & $\stackrel{5}{0}$ & $\begin{array}{l}0 \\
+ \\
0\end{array}$ & & $\begin{array}{l}0 \\
\text { ñ. } \\
0\end{array}$ & 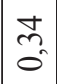 & $\left|\begin{array}{c}\infty \\
\infty \\
0 \\
0\end{array}\right|$ & $\left|\begin{array}{c}\infty \\
\multirow{2}{2}{} \\
0\end{array}\right|$ & $\dot{\sigma}_{0}$ & $\stackrel{⿱}{\tilde{\sigma}}$ & $\dot{\sigma}_{0}$ & $\mid \begin{array}{l}0 \\
0 \\
0 \\
0\end{array}$ & $\begin{array}{l}J_{0} \\
0\end{array}$ & $\mid$ & $\vec{\sigma}$ & ?) & $\begin{array}{l}0 \\
0 \\
0\end{array}$ & $\stackrel{2}{2}$ & $\begin{array}{c}\tilde{n} \\
\tilde{0}\end{array}$ & $\stackrel{n}{2}$ \\
\hline$\tilde{U}$ & fr & $\stackrel{m}{\tilde{0}}$ & $\frac{9}{0}$ & & ñ? & $\overrightarrow{\sigma_{2}}$ & $\begin{array}{l}\hat{y} \\
\hat{0}\end{array}$ & 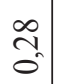 & సి- & $\begin{array}{l}0 \\
2 \\
0 \\
0\end{array}$ & $\begin{array}{l}\vec{\lambda} \\
0\end{array}$ & $\left|\begin{array}{l}0 \\
\multirow{2}{*}{} \\
0\end{array}\right|$ & $\begin{array}{l}0 \\
=\end{array}$ & $\frac{1}{0}$ & $\begin{array}{l}8 \\
\dot{0} \\
0\end{array}$ & o. & $\begin{array}{l}\because \\
0\end{array}$ & 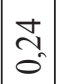 & $\begin{array}{l}n \\
\tilde{2} \\
0\end{array}$ & - \\
\hline$a^{\circ}$ & $\cong$ & $=$ & $\stackrel{\Xi}{\simeq}$ & & $\hat{0}$ & 의 & $\begin{array}{l}0 \\
0 \\
a\end{array} \mid$ & $\stackrel{+}{\dot{\sigma}_{0}}$ & 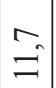 & $\cong$ & $\begin{array}{l}0 \\
\therefore \\
n\end{array} \mid$ & $\begin{array}{l}\stackrel{+}{ \pm} \\
\stackrel{ \pm}{*}\end{array}$ & $\left|\begin{array}{l}0 \\
\infty \\
-\end{array}\right|$ & $\underset{\sim}{\Xi}$ & ㄷ & 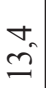 & $\frac{7}{\infty^{\circ}}$ & $\overrightarrow{\vec{Z}^{\prime}}$ & $a$ & $\Xi$ \\
\hline 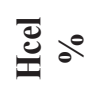 & $\begin{array}{l}\sim \\
\stackrel{\sim}{\sim}\end{array}$ & ले & $\hat{m}$ & & $\stackrel{\text { ¿े }}{0}$ & $\begin{array}{l}0 \\
\dot{m} \\
\dot{m}\end{array}$ & $\begin{array}{l}0 \\
\stackrel{i}{2}\end{array}$ & $\begin{array}{l}\infty \\
\dot{m} \\
\dot{m}\end{array}$ & $\mid$ & min & $m$ & $\overrightarrow{\tilde{e}^{\prime}}$ & $=$ & $\left|\begin{array}{l}\infty \\
\tilde{d} \\
\text { in }\end{array}\right|$ & $\overrightarrow{\tilde{n}}$ & बे & $\left|\begin{array}{l}\infty \\
0 \\
0 \\
\tilde{n}\end{array}\right|$ & $\mid \begin{array}{c}n \\
\tilde{n} \\
m\end{array}$ & 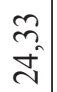 & i \\
\hline$\dot{\vec{e}} 0^{\circ}$ & 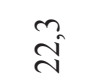 & $\stackrel{\sim}{\sim}$ & ते & & $\hat{\vec{v}}$ & $\begin{array}{l}n \\
\tilde{n} \\
\end{array}$ & $\begin{array}{l}a \\
\dot{v}\end{array}$ & $\begin{array}{l}\hat{\sigma} \\
\hat{\sim}\end{array} \mid$ & $\left|\begin{array}{l}0 \\
\dot{\sim}\end{array}\right|$ & $\vec{d}$ & $\begin{array}{l}\infty \\
\hat{\sim} \\
0\end{array}$ & $\vec{\sim}$ & $\left|\begin{array}{l}\infty \\
= \\
=\end{array}\right|$ & ते & $\Xi$ & $\hat{\tilde{i}}$ & $\begin{array}{l}\tilde{n} \\
\tilde{n}\end{array}$ & $\mid$\begin{tabular}{l}
0 \\
\multirow{2}{*}{+1} \\
-1 \\
-1
\end{tabular} & $\infty$ & 이 \\
\hline$\underbrace{000}$ & ఫू & in & $\frac{7}{7}$ & & ơ & $\begin{array}{l}0 \\
0 \\
\end{array}$ & $\frac{2}{2}$ & $\begin{array}{l}2 \\
\infty \\
-\end{array} \mid$ & $\tilde{\Omega}$ & \begin{tabular}{l}
$\infty$ \\
\multirow{\sigma}{*}{}
\end{tabular} & $\underset{⿱}{\stackrel{⿱}{\Im}}$ & $\begin{array}{l}\stackrel{+}{\sim} \\
\dot{\nabla}\end{array}$ & $\stackrel{n}{=}$ & $\left|\begin{array}{l}0 \\
\multirow{\sigma}{*}{}\end{array}\right|$ & $\begin{array}{c}\infty \\
\infty \\
m\end{array}$ & $\stackrel{m}{\vec{\sigma}}$ & 点 & $\frac{\approx}{n}$ & 6 & $m$ \\
\hline 穴。 & ڤn & $\hat{\tilde{e}}$ & กิ & & $\frac{n}{m}$ & 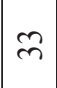 & $\left|\begin{array}{c}\tilde{a} \\
\tilde{n} \\
\tilde{n}\end{array}\right|$ & $\begin{array}{l}\hat{n} \\
\tilde{n}\end{array}$ & $\begin{array}{l}\tilde{m} \\
\tilde{m}\end{array}$ & ते & 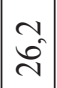 & $\left|\begin{array}{l}n \\
0 \\
\tilde{N}^{2}\end{array}\right|$ & $\hat{\sim}^{2}$ & $\left|\begin{array}{c}\overrightarrow{0} \\
\infty \\
\sim\end{array}\right|$ & $\overrightarrow{\vec{v}}$ & in & $\begin{array}{c}0 \\
0 \\
\infty \\
\infty \\
0\end{array} \mid$ & 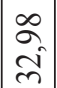 & $\begin{array}{l}\tilde{b} \\
\tilde{\sigma}\end{array}$ & $\begin{array}{l}n \\
\approx \\
\tilde{a}\end{array}$ \\
\hline 貟。 & 巽 & fं & 8 & $\begin{array}{l}0 \\
\text { is }\end{array}$ & $\approx$ & $\begin{array}{l}\text { ?. } \\
8\end{array}$ & $\mid \begin{array}{l}\infty \\
6\end{array}$ & $\hat{8}$ & $\hat{\tilde{n}}$ & बิ & $\begin{array}{l}n \\
\tilde{n} \\
n\end{array}$ & $\left|\begin{array}{l}0 \\
0 \\
0 \\
n\end{array}\right|$ & $\begin{array}{c}m \\
\hat{q}\end{array}$ & $\frac{n}{n}$ & $\begin{array}{c}m \\
\hat{n}\end{array}$ & $\stackrel{\circ}{n}$ & $\begin{array}{l}\tilde{\lambda} \\
\ddot{2}\end{array}$ & $\mid \begin{array}{l}\widetilde{1} \\
8 \\
8\end{array}$ & 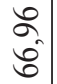 & $\begin{array}{l}\infty \\
\infty \\
\infty \\
m\end{array}$ \\
\hline 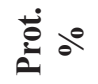 & $\tilde{\sigma}^{2}$ & r & î & & $\stackrel{+}{n}$ & ఫُ & 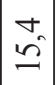 & $\tilde{\sigma}^{\circ}$ & $\begin{array}{l}\infty \\
\stackrel{-}{二}\end{array}$ & $\begin{array}{l}2 \\
\infty\end{array}$ & $\overrightarrow{\mathrm{v}}$ & $\left|\begin{array}{l}\infty \\
\hat{\imath} \\
\hat{\sim}\end{array}\right|$ & $\left|\begin{array}{|}\infty \\
\hat{\lambda}\end{array}\right|$ & $\begin{array}{l}\infty \\
0 \\
0\end{array}$ & $\overrightarrow{\tilde{\lambda}}$ & $\vec{\sim}$ & $\stackrel{\sim}{\underset{J}{ \pm}}$ & 0 & $\stackrel{\tilde{g}}{=}$ & 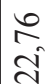 \\
\hline 됟 & i) & శึ & & & & ju & $\begin{array}{l}2 \\
0 \\
i\end{array} \mid$ & $\begin{array}{l}n \\
i\end{array}$ & $\begin{array}{l}\text { vo } \\
\text { i }\end{array}$ & $\frac{I}{m}$ & $\frac{n}{m}$ & $\left|\begin{array}{l}\infty \\
n \\
n \\
m\end{array}\right|$ & $\left|\begin{array}{c}5 \\
0 \\
\dot{\sigma}\end{array}\right|$ & $\left|\begin{array}{l}\overrightarrow{0} \\
0 \\
i\end{array}\right|$ & $\begin{array}{l}\underset{f}{ \pm} \\
\underset{f}{*}\end{array}$ & $m$ & $\begin{array}{l}\tilde{\alpha} \\
\tilde{i}\end{array}$ & $\left|\begin{array}{l}0 \\
0 \\
i\end{array}\right|$ & $\begin{array}{l}J_{0} \\
\text { m. }\end{array}$ & $a$ \\
\hline$\sum^{\infty} a^{0}$ & $\stackrel{\sigma}{\sigma}$ & o & & s & 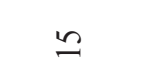 & 0 & $\vec{\infty}$ & $\hat{\tilde{d}}$ & $\overrightarrow{=}$ & $\begin{array}{l}\sigma \\
\infty\end{array}$ & $\because$ & $\vec{m}$ & $\begin{array}{l}0 \\
0 \\
0\end{array}$ & $\vec{m}$ & $\begin{array}{l}\stackrel{+}{ \pm} \\
\stackrel{ \pm}{*}\end{array}$ & ֶָ, & n̊. & 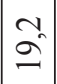 & $\begin{array}{c}\infty \\
\approx \\
\infty \\
\sim\end{array} \mid$ & $\begin{array}{l}\circ \\
\stackrel{0}{0} \\
\text { లె }\end{array}$ \\
\hline $\pm 0^{\circ}$ & $\stackrel{0}{0}$ & $\begin{array}{l}\infty \\
\infty \\
\infty\end{array}$ & $\hat{\infty}$ & ó & 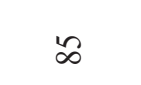 & $\frac{0}{\infty}$ & $\frac{\sigma}{\infty}$ & $\stackrel{m}{\stackrel{2}{R}}$ & $\begin{array}{l}\hat{\text { aे }} \\
\infty\end{array}$ & $\vec{\infty}$ & $\infty$ & $\left|\begin{array}{l}\hat{2} \\
\infty \\
\infty\end{array}\right|$ & $\begin{array}{l}\overrightarrow{+} \\
\stackrel{\infty}{\infty}\end{array}$ & $\left|\begin{array}{l}\hat{\sigma} \\
\dot{\infty}\end{array}\right|$ & $\begin{array}{c}0 \\
\vdots \\
\infty \\
\infty\end{array}$ & $\begin{array}{c}\infty \\
\infty \\
\infty\end{array}$ & $\hat{2}$ & $\begin{array}{l}\infty \\
0 \\
\infty\end{array}$ & $\begin{array}{l}\tilde{Z} \\
- \\
-\end{array}$ & $\begin{array}{l}\text { D } \\
\text { తి }\end{array}$ \\
\hline 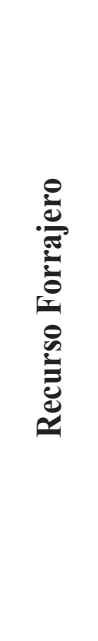 & 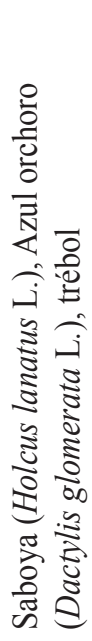 & 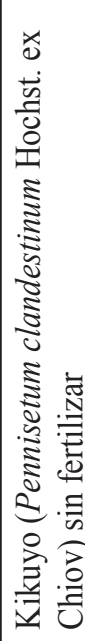 & 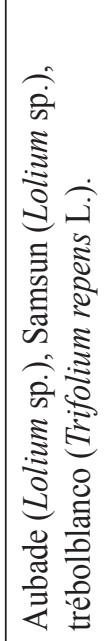 & 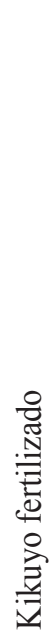 & 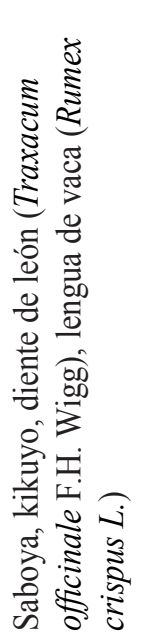 & 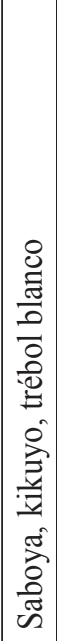 & 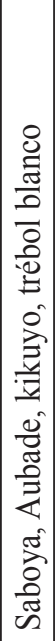 & 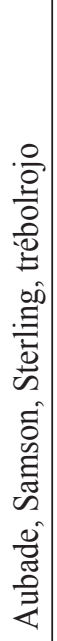 & 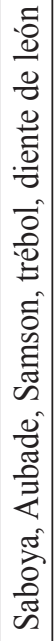 & 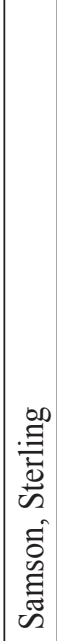 & 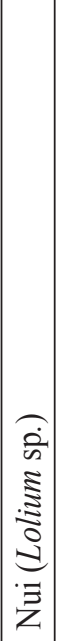 & 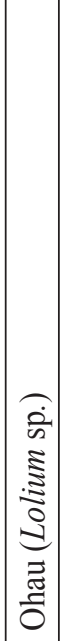 & 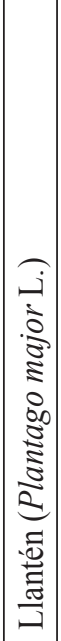 & 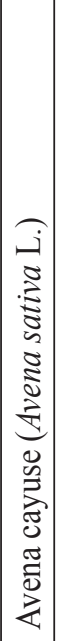 & 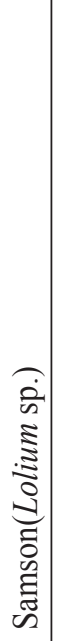 & 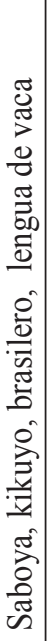 & $\mid$ & 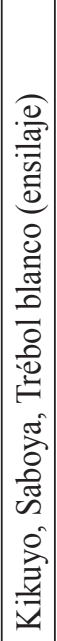 & 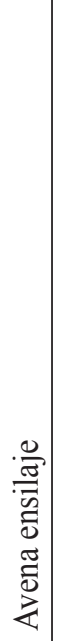 & 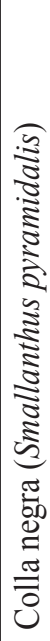 \\
\hline 을 & & & & $\sigma$ & & 6 & - & $\infty$ & $a$ & 잉 & $=$ & $\simeq$ & $\Omega$ & \pm & $\because$ & 0 & 二 & $\infty$ & 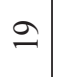 & \\
\hline
\end{tabular}


Belalcázar y Návaez (2008) aseveran que "el contenido de proteína de algunos follajes de arbóreas es superior al de las gramíneas", como en este caso la colla negra.

Se observó además, una correlación negativa muy baja $(-0,02406)$ entre el porcentaje de proteína y la producción total de $\mathrm{CH}_{4}$ (Fig. 1).

\section{Fibra Detergente Neutra (FDN)}

Los contenidos de fibra detergente neutra no reportaron diferencias estadísticas importantes $(p>0,05)$. El ensilaje de avena fue la dieta con el porcentaje más elevado para esta variable, con un valor cercano a los reportados por Calsamiglia et al. (2005), quienes reportan valores de FDN para el ensilaje de avena de 67,6\%; así mismo, la Fundación Española para el Desarrollo de la Nutrición Animal (FEDNA) reporta valores de $59,56 \%$ para FDN para la misma especie en ensilaje (Tab. 3).

Los resultados obtenidos para esta variable presentaron una correlación negativa muy baja $(-0,03891)$ con respecto a la producción total de gas metano.

\section{Fibra Detergente Acida (FDA)}

No se presentaron diferencias estadísticas $(\mathrm{p}>0,05)$ para la fibra detergente acida (FDA) (Tab. 3). El menor valor numérico para esta variable se obtuvo con el pasto Samson.

En general, los valores encontrados están por debajo de los reportados por el ICA (1994), quien afirma que los valores cercanos al $40 \%$ son considerados como altos. Se presentó además, una correlación negativa muy baja $(-0,15954)$ con respecto a los valores de producción de gas metano y una correlación negativa baja $(-0,25298)$ con los ácidos grasos volátiles (AGV's).

\section{Producción de gas de las especies forrajeras evaluadas}

La Figura 1 muestra los valores de producción de $\mathrm{CH}_{4}$ obtenidos para cada dieta. La mezcla de saboya, aubade, kikuyo y trébol blanco presentó el nivel más alto de producción, seguido por Avena cayuse, Samson y la mezcla con saboya, kikuyo, brasilero y lengua de vaca.

Los valores encontrados pueden estar relacionados con el estado fenológico de las plantas, ya que puede ser decisiva al momento de relacionarla con emisiones significativas; a medida que aumentó la madurez se observó mayor producción de metano, por ejemplo, periodos de recuperación prolongados observados en Pennisetum clandestinum, la mezcla de éste con Holcus lanatus o con Phalaris sp., con mayor concentración de fibra y materia seca, emitieron más metano al medio ambiente,razón por la cual, si el objetivo es reducir la emisión de gases sin afectar la calidad nutricional de la pradera, es aconsejable suministrar dietas con plantasen estados óptimos de cosecha.

En este sentido, Montenegro y Abarca (2000) afirman que existen evidencias que relacionan la tasa de emisión de metano por fermentación entérica con el tipo de alimento consumido y las características físicas y químicas del mismo, las cuales afectan directamente el nivel de consumo y la frecuencia de alimentación. Por tanto, una subnutrición causada por una mala calidad del alimento, podría contribuir a incrementar los niveles de emisión de metano.

Watson et al. (1996) afirman que las emisiones de $\mathrm{CH}_{4}$ por unidad de alimento consumido pueden reducirse si se utilizan sistemas de pastoreo eficientes y un pasto de mejor calidad, pues los animales que pastan en tierras de mala calidad producen más $\mathrm{CH}_{4}$ por unidad de alimento consumido. 


\section{Ecuación de regresión entre presión y volumen}

Con los datos de presión y volumen obtenidos en la investigación, derivados del proceso de la fermentación en los diferentes horarios de incubación, se construyó una ecuación de regresión a partir de la relación entre presión y volumen (Tab. 4), para determinar las características de presión atmosférica y condiciones ambientales del Laboratorio de Ciencias Pecuarias de la Universidad de Nariño, en la ciudad de Pasto, Colombia, para el desarrollo de la técnica in vitro de producción de gasespara posteriores estudios.

Al relacionar los resultados obtenidos entre la presión y el volumen del presente estudio, con los obtenidos en centros de investigación ubicados en diferentes pisos altitudinales, se confir- ma lo enunciado por Posada y Noguera (2005), quienes afirman que el aumento de presión atmosférica conserva una relación lineal con el volumen de gas estimado desde las ecuaciones obtenidas, evento confirmado anteriormente por Narváez y Delgado (2011) y reiterado nuevamente en la presente investigación.

\section{Ácidos GrasosVolátiles (AGV’S)}

El análisis estadístico reveló que no existen diferencias significativas para la producción de AGV's ( $p>0,05)$ entre dietas (Fig. 2); sin embargo, se obtuvo los mayores valores de ácido propiónico en el llantén, Samson, y la mezcla de Saboya, Aubade, kikuyo y trébol blanco. Estos resultados pueden relacionarse con relevantes contenidos de celulosa y lignina presentes en las especies evaluadas.

Tabla 4. Ecuación de regresión entre presión (psi) (X) y volumen de gas (ml) (Y) obtenida en el presente estudio. Laboratorios de Ciencias Pecuarias - Universidad de Nariño

\begin{tabular}{ccccc}
\hline Inóculo & $\begin{array}{c}\text { Altitud } \\
(\mathbf{M s n m})\end{array}$ & Ecuación & R2 & $\begin{array}{c}\text { Relación } \\
\text { Presión: Volumen }\end{array}$ \\
\hline Inóculo fecal & 2488 & $\mathrm{Y}=-0,024+5,531 \mathrm{X}+0,0048 \mathrm{X}^{2}$ & 0,99 & $1 \mathrm{psi}=5,51 \mathrm{ml}$ \\
\hline
\end{tabular}

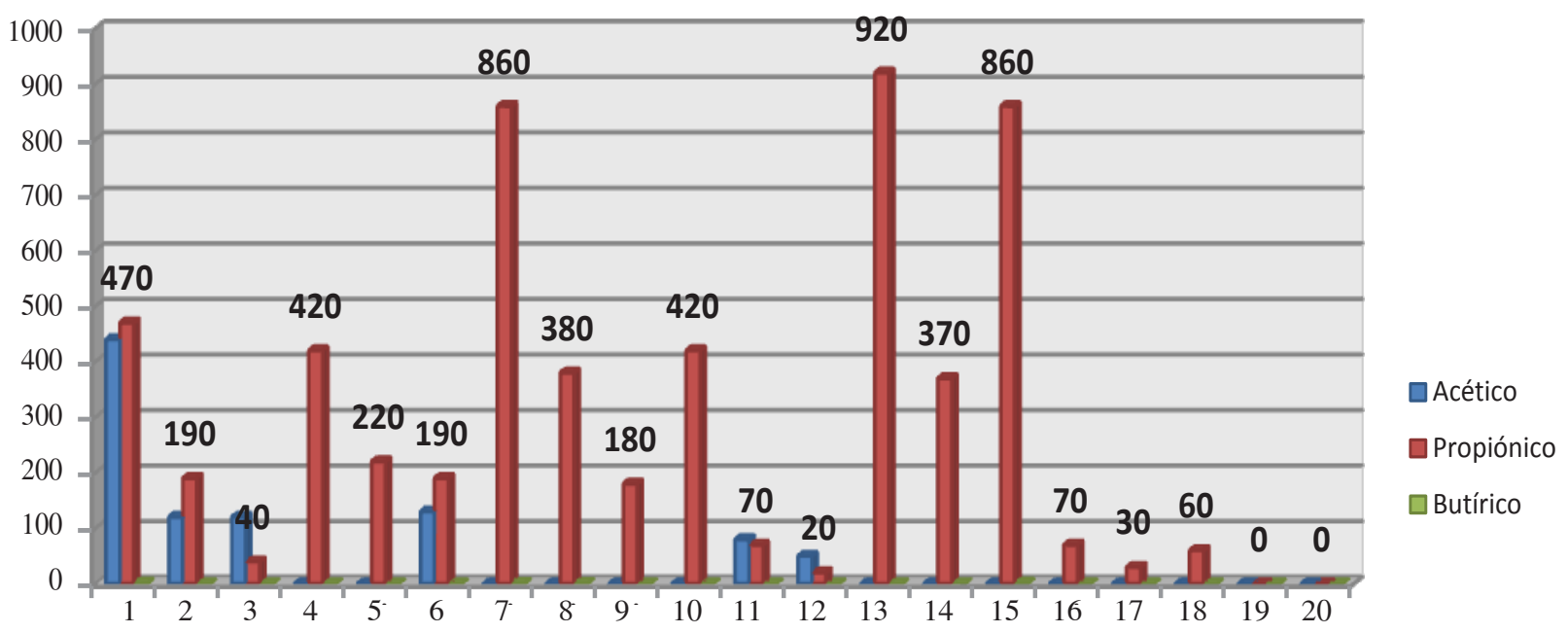

Figura 2. Producción de AGV's de los recursos forrajeros evaluados, listados en la Tabla 3 
La pradera en mezcla de saboya, azul orchoro y trébol rojo, presentó el valor más alto de ácidoacético, con $470 \mathrm{ml} / \mathrm{L}$, tratamiento con altos contenidos de fibra. Álvarez (2000) afirma que la mayor producción de AGV's, proviene de las fracciones de fibra de los alimentos, componentes que incrementan la producción de acetato en los forrajes. La determinación de ácido butírico, no presentó valores significativos en ninguna de las praderas evaluadas, sin embargo, estos valores no influyen en la calidad nutricional de degradabilidad de la materia seca (DMS) y la materia orgánica (DMO).

La degradación de la materia seca y materia orgánica (Fig. 3 y 4 ) presentó diferencias $(p<0,05)$, con valores altos de DMS para Avena cayuse, y la mezcla de Aubade, Samson y trébol blanco presentó el valor más bajo.

Por otro parte, la DMO presentó su mayor porcentaje en la mezcla de Kikuyo, Saboya y trébol blanco, y llantén.

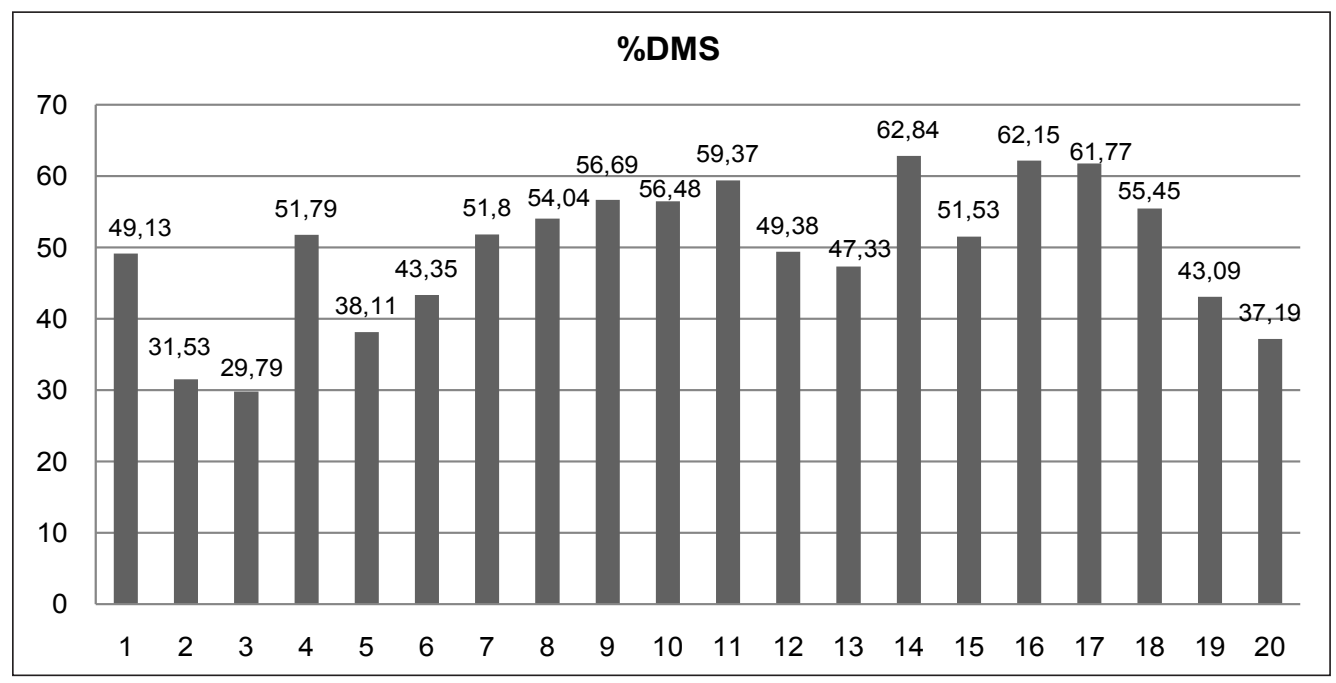

Figura 3. Degradabilidad de la materia seca (DMS)de los recursos forrajeros evaluados, listados en la Tabla 3

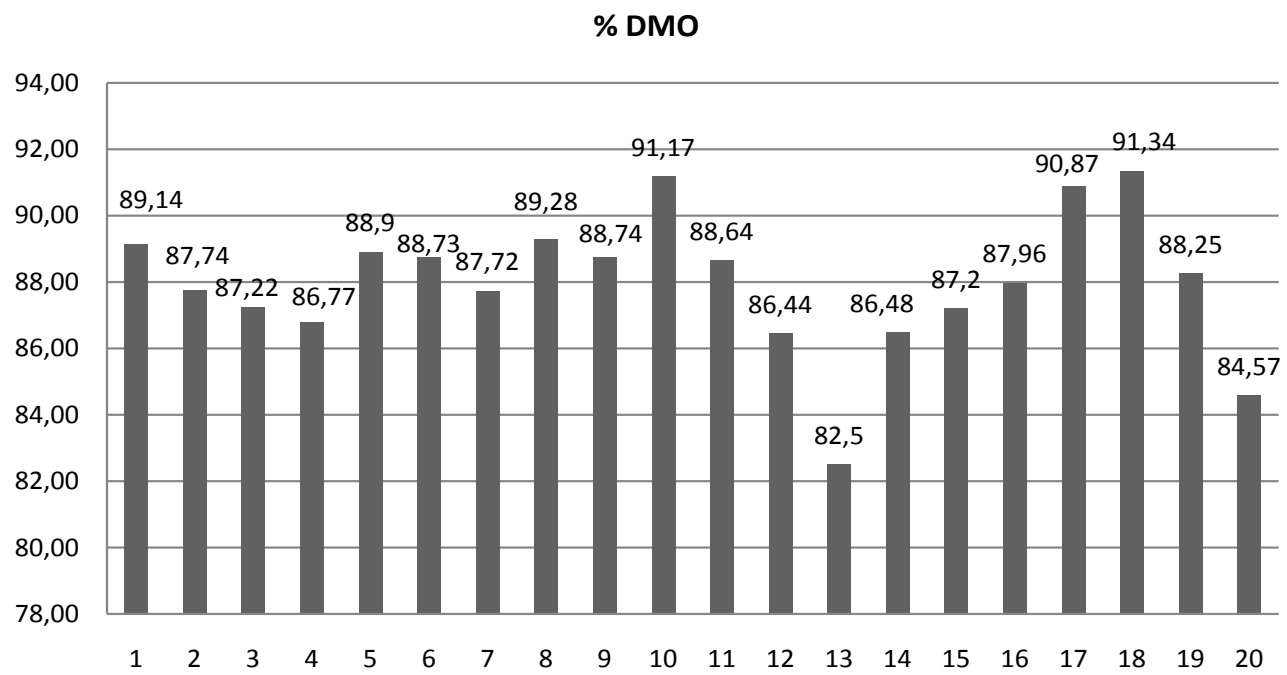

Figura 4. Degradabilidad in vitro de materia orgánica (DMO) de los recursos forrajeros evaluados, listados en la Tabla 3 
Los porcentajes en $\mathrm{DMO}$, permiten afirmar que las plantas de rápido crecimiento como los Lolium sp. y otras gramíneas con mayor contenido de carbohidratos solubles, de fácil aprovechamiento por los microorganismos y sistema digestivo de animales, aminoran la emisión de gases efecto invernadero.

\section{CONCLUSIONES}

La técnica in vitro de producción de gases es un procedimiento confiable para determinar la emisión de gases efecto invernadero en condiciones de trópico elevado, de tal manera que queda abierta la posibilidad de realizar futuras investigaciones.

El desarrollo, adecuación y aplicación de la técnica in vitro de producción de gases, bajo las condiciones del laboratorio de la Universidad de Nariño, permitió establecer una ecuación de regresión aplicable a futuros estudios.

La pradera en mezcla de Saboya, Aubade, kikuyo y trébol blanco presentó un elevado potencial de producción de gas metano $\left(\mathrm{CH}_{4}\right)$, expresado por sus bajos contenidos de proteína.

La calidad de los recursos forrajeros que se suministra a los rumiantes es determinante sobre la digestibilidad, la producción de AGVs y las emisiones de Gases Efecto Invernadero como el gas metano $\left(\mathrm{CH}_{4}\right)$.

Es importante tener en cuenta el suministro de praderas en óptimos estados fisiológicos, jóvenes, sinónimo de mayor calidad nutricional y menores pérdidas energéticas a nivel ruminal a causa de la producción de metano.

En general, la asociación entre gramíneas, leguminosas y arvenses presenta menores producciones de gas metano.

\section{BIBLIOGRAFÍA}

ÁLVAREZ, D.M.2000. Evaluación in vitro de leguminosas tropicales como fuente de proteína para rumiantes. Trabajo de grado para optar al título de Zootecnista. Palmira. Universidad Nacional de Colombia. 120 p.

BELALCAZAR, L. y NARVÁEZ, O.2008. Valoración nutritiva del forraje colla negra (Smallanthus pyramidalis) en mezcla con pato kikuyo (Pennisetum clandestinum) en la alimentación de cuyes (Cavia porcellus), fases de levante y engorde. Trabajo de grado para optar al título de Zootecnista. Pasto Nariño. Universidad de Nariño. 65p.

BERRA, G., FINSTER, L., CASTUMA, E. y MALDONADO, V. 2006. Reducción y opciones de mitigación de emisiones de metano - ganado bovino. Ministerio de Desarrollo Social y Medio Ambiente. Secretaría de Desarrollo Sustentable y Política Ambiental. Buenos Aires, Argentina. $142 \mathrm{p}$.

CALSAMIGLIA, S., CASTILLEJOS, L. y BUSQUET, M. 2005. Federación Española para el Desarrollo de la Nutrición Animal - FEDNA. Estrategias nutricionales para modificar la fermentación ruminal en vacuno. Madrid, 171 p.

FOOD AND AGRICULTURE ORGANIZATION OF THE UNITED NATIONS (FAO). 2006. Las repercusiones del ganado en el medio ambiente. El desafío estriba en reconciliar dos demandas: La de productos animales y la de servicios ambientales. En: http://www.fao.org/ag/esp/ revista/0612sp1.htm.; consultado: octubre, 2014.

GOERING, H.K. y VAN SOEST, P.J. 1970. Forage fiber analysis (apparatus, reagents, procedures, and some applications). Agriculture Handbook. No. 379. Agricultural Research Service, Washington, D.C. 20 p. 
HOLDRIDGE, L.E. 1978. Ecología basada en zonas de vida. Instituto Interamericano de Ciencias Agrícolas. San José, Costa Rica. 215 p.

INSTITUTO COLOMBIANO AGROPECUARIO (ICA). 1994. Programa de pastos y forrajes.

MONTENEGRO, J. y ABARCA, S. 2000. Fijación de carbono, emisión de metano y de óxido nitroso en sistemas de producción bovina en Costa Rica. En: Intensificación de la ganadería en Centroamérica: beneficios económicos y ambientales. CATIE - FAO - SIDE. Ed Nuestra Tierra. 334 p.

NARVÁEZ, J. y DELGADO, J. 2011. Caracterización de recursos forrajeros herbáceos, arbóreos y arbustivos de uso convencional y alternativo en el trópico alto del departamento de Nariño, mediante el uso de la Técnica in vitro de producción de gases. Universidad de Nariño. Facultad de Ciencias Pecuarias. Programa de Zootecnia. Tesis de grado para optar al título de Zootecnista.

OFFICIAL METHODSOF ANALYSIS(A.O.A.C). 1990. Officialmethods of analysis. $15^{\text {th }}$ edn. Association of official analytical chemists. 771 p.

PELL, A.N., DOANE P.H.y SCHOFIELD, P. 1997. In vitro digestibility and gas production. In: Simpósio sobre Tópicos Especiais em Zootecnia, Lavras, MG.109 - 132 p.

POSADA, S.L. y NOGUERA R.R. 2005. Técnica in vitro de producción de gases: Una herramienta para la evaluación de alimentos para rumiantes. Livestock Research for Rural Development. 17(4).

POSADA S.L, y NOGUERA R.R. 2005. En: Livestock Research for Rural Development. En: http:/ / www.cipav.org.co/lrrd/lrrd17/4/posa17036.htm; consulta: marzo, 2010.
SIERRA, J. 2006. Monitoreo o aforo del rendimiento de forraje para el cálculo de la carga animal en sistemas de pastoreo racional y el ajuste de carga en pesajes intermedios.

SMITH, V.N. 1993. Profesor de Genética y Mejoramiento Forestal. Universidad de Talca. Santiago de Chile. En metodologías para el muestreo para la selección de árboles. En: http://www. cesaf.uchile.cl/cesaf/n2/indice.htm; consulta: junio, 2010.

TILLEY, JMA. y TERRY, RA. 1963. A two-stage technique for the in vitro digestion of forage crops. Journal of the British Grassland Society. 18:104 - 111.

THEODORIDOU, K., AUFRÈRE, J., ANDUEZA, D., LE MORVAN, A., PICARD, F., POURRAT, J. y BAUMONT, R. 2012. Effects of condensed tannins in wrapped silage bales of sainfoin (Onobrychis viciifolia) on in vivo and in situ digestion in sheep. Animal 6: $245-253$

WATSON, R.T., ZINYOWERA, C.M. y MOSS, R.H. 1996. Tecnologías, políticas y medidas para mitigar el cambio climático: Documento técnico I del IPCC. 87 p. 